Accretion Phenomena and Related Outflows, IAU Colloquium 163

ASP Conference Series, Vol. 121, 1997

D.T. Wickramasinghe, L. Ferrario, and G.V. Bicknell, eds.

\title{
General Relativistic Simulation of Jet Formation from Magnetized Accretion Disk
}

\author{
Shinji Koide \\ Faculty of Engineering, Toyama University, Gofuku, Toyama 930, Japan \\ Kazunari Shibata, Takahiro Kudoh \\ National Astromonical Observatory, Mitaka, Tokyo 181, Japan
}

\begin{abstract}
Recently, superluminal motions are observed not only from active galactic nuclei but also in our Galaxy. These phenomena are explained as relativistic jets propagating almost toward us with Lorentz factor more than 2. For the formation of such a relativistic jet, magnetically driven mechanism around a black hole is most promising. We have extended the 2.5D Newtonian MHD jet model (Shibata \& Uchida 1986) to general relativistic regime. For this purpose, we have developed a general relativistic magnetohydrodynamic (GRMHD) numerical code and applied it to the simulation of the magnetized accretion disk around a black hole. We have found the formation of magnetically driven jets with 86 percent of light velocity (i.e. Lorentz factor $\sim 2.0$ ).
\end{abstract}

\section{Introduction}

Observations with VLBI and VLA have revealed radio jets not only from quasars and active galactic nuclei but also from the 'microquasars' in our Galaxy such as GRS $1915+105$ and GRO J1655-40, parts of which sometimes appear to move faster than light (Miley 1980, Mirabel \& Rodriguez 1994). These superluminal motions are explained by relativistic jets with small inclination angle between the direction of the motion of the jet and the line of sight. These relativistic jets are thought to arise from processes associated with black holes. Blandford \& Payne (1983) proposed a magnetically driven jet model for the jet ejected from accretion disks. This model is extended to relativistic regime using analytical methods (e.g. Cammenzind 1989, Li, Chiueh \& Begelman 1992, Tomimatsu 1995). On the other hand, Shibata \& Uchida (1986) showed the formation of a magnetically driven jet from the accretion disk, by using nonrelativistic, nonsteady 2.5D MHD simulations. In their model, a dense, cold isothermal gas disk is initially rotating around the central object with a (sub-)Keplerian velocity and the initial magnetic field is assumed to be uniform and parallel to the rotation axis of the disk. As the rotating disk contracts, it pulls the magnetic field towards the center as well as the azimuthal direction. The sandglass shaped magnetic surface is formed and magnetic twist accumulates. The plasma in the surface layers of the disk is accelerated to the polar directions by magnetic 
(a)

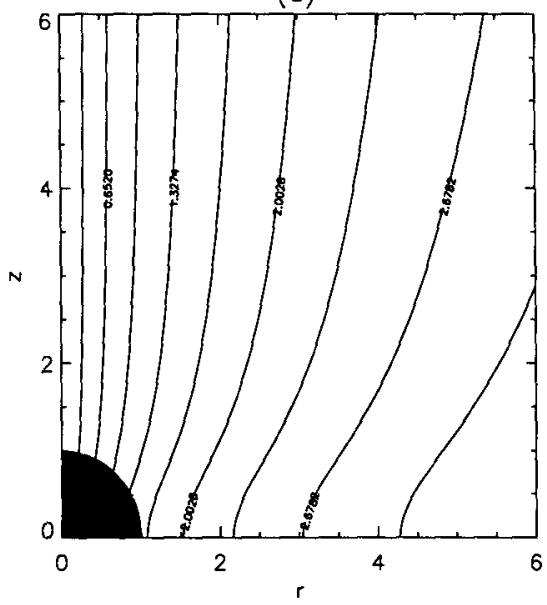

(b)

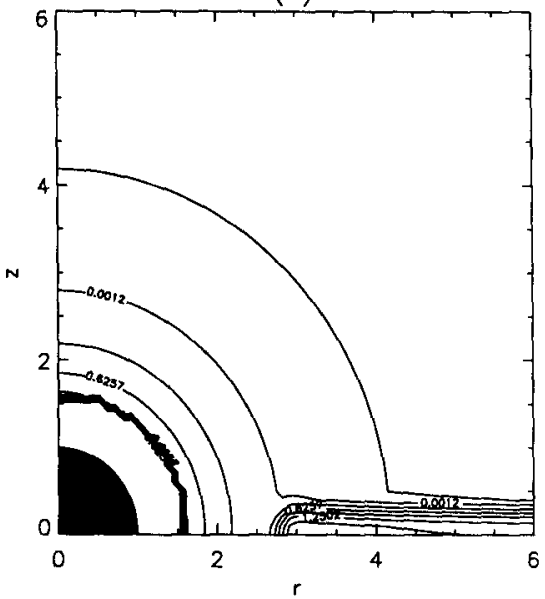

Figure 1. Initial state of GRMHD calculation of relativistic jet formation around a black hole. Panels (a) and (b) show magnetic field lines and mass density, respectively. The black region at the origin indicates a black hole whose surface is the Schwarzschild radius.

pressure and centrifugal force. The accelerated gas is collimated by the magnetic field and forms a supersonic bipolar jet.

In this paper, we report on the first full numerical simulation of the magnetically driven relativistic jet from the accretion disk around a black hole which is an extension of the Newtonian MHD jet model (Shibata \& Uchida 1986) to general relativistic regime. We have developed a general relativistic magnetohydrodynamic (GRMHD) code and applied it to simulate the jet. We have succeeded to form a relativistic jet with 86 percent of light velocity.

\section{Numerical Method and Results}

We use GRMHD equations with the frozen-in condition. Here we assume that the off-diagonal elements of the metric vanish. In this framework, we use Schwarzschild metric. We employ the simplified total variation diminishing (STVD) method (Koide et al 1996a). We applied the special relativistic version of this code to the propagation of relativistic magnetized jet successfully (Koide et al 1996a, Koide 1996b).

Figures 1 and 2 show the results of numerical simulations of the relativistic jet formation. We perform the simulation with Schwarzschild metric in the box $0 \leq r \leq 10,0 \leq z \leq 10$, with $100 \times 100$ meshes, assuming the axisymmetry with respect to $z$-axis and the mirror symmetry with respect to the plane $z=0$. These figures show the part of the simulation box: $0 \leq r \leq 6,0 \leq z \leq 6$. The initial magnetic field and mass density are shown in Figs. $1(\mathrm{a})$ and $1(\bar{b})$, respectively. 
(a)

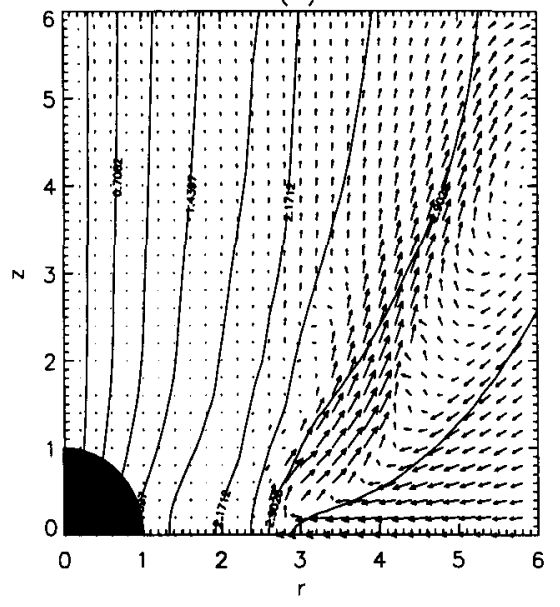

(b)

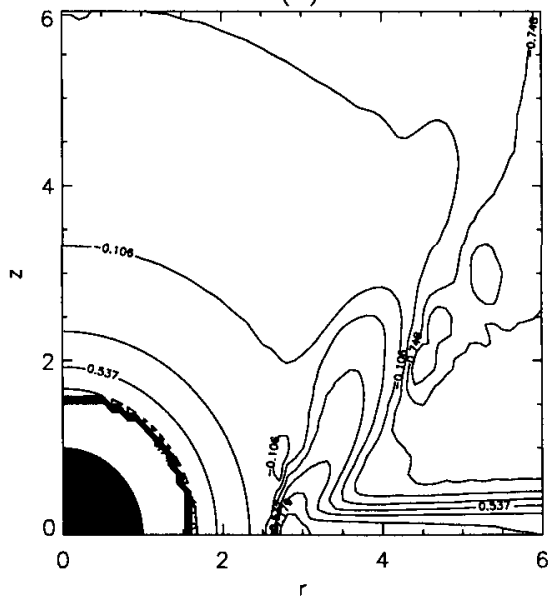

Figure 2. The snapshot at $t=47.4 \tau_{\mathrm{K}}$. (a) velocity and magnetic field. (b) logarithm of density.

The initial state of the simulation is divided into two parts: a background corona around a black hole and an accretion disk. The black region in theses figures show the black hole with the radius $r_{\mathrm{s}}$ at the center. Here $r_{\mathrm{s}}$ is Schwarzschild radius and is defined as $r_{\mathrm{s}} \equiv 2 G M / c^{2}$, where $G$ and $M$ is gravitational constant and the mass of the compact object. The Schwarzschild radius $r_{\mathbf{s}}$ is used as unit of length. In the corona, plasmas are assumed to be isothermal and in hydrostatic equilibrium. Here the sound velocity is constant $(0.1 c)$. At the Schwarzschild radius, not only the metric but also the density and the pressure of the corona diverge. Near the horizon, numerical dissipation due to STVD scheme becomes significant. We set damping region at $1.8 \leq r \leq 2.5$ to avoid the numerical instability. The accretion disk is located at $z=0, \bar{r} \geq r_{\mathrm{D}} \equiv 2.8 r_{\mathrm{s}}$ with its thickness $\delta=0.25 r_{\mathrm{s}}$ and rotates around the black hole with almost Keplerian velocity $v_{\mathrm{K}}$. Inside the spherical surface $r \leq 3 r_{\mathrm{s}}$, there in no stable Keplerian orbit around the black hole. In this case, the rotational velocity of the disk is 60 percent of light velocity at its inner edge. The mass density is hundred times of that of the background corona (Fig. 1b). In addition, the magnetic field crosses the accretion disk perpendicularly (Fig. 1a). To speed up the calculation, we employ the sandglass shaped poloidal magnetic field as the initial condition of the magnetic field (Cao \& Spruit 1994). At the point of the accretion disk $\left(r=3 r_{\mathbf{s}}, z=0\right)$, the Alfvén velocity is $v_{\mathrm{A}}=0.1 c$.

Fig. 2 shows the velocity, magnetic field, and mass density at $t=47.4 \tau_{\mathrm{K}}$. Here $\tau_{\mathrm{K}}$ is defined as $\tau_{\mathrm{K}} \equiv r_{\mathrm{s}} / c$. The jet is ejected from near the inner edge of the disk (Fig. 2a). The jet has a hollow cylindrical shell structure with helical motion in it as in the nonrelativistic case (Shibata \& Uchida 1986). The jet is accelerated especially at the point $r=3.6 r_{\mathrm{s}}, z=1.5 r_{\mathrm{s}}$ where the inclination angle of the magnetic surface is less than $60^{\circ}$ as pointed by Cao and Spruit 
(1994). Near the point, the maximum velocity of the jet is 86 percent of light velocity which corresponds Lorentz factor 2.0 . The maximum jet velocity is $\sim 1.5 v_{\mathrm{K}}\left(r \sim r_{\mathrm{D}}\right)$. This factor is in agreement with nonrelativistic theory by Kudoh and Shibata (1995) when the ambient magnetic field is strong enough. We can see the dense plasma is ejected to form the relativistic jet (Fig. 2b). Our result shows that, even in relativistic region, the velocity of the magnetically driven jet is order of Keplerian velocity as reported by Kudoh \& Shibata (1995) for the nonrelativistic analysis. In this stage, the poloidal component of the maximum velocity is 25 percent of light velocity. However, when the knot of the jet propagates far from the core region, the direction of the jet changes along the magnetic surface and the polar velocity component of the jet is expected to be the total velocity at this stage: $v_{\max } \sim 0.85 c$.

Mirabel \& Rodriguez (1994b) showed that the velocity of the ejecta from black hole candidates such as GRS $1915+105$ and GRO J1655-40 is larger than $0.9 \mathrm{c}$ whereas it is less equal to $0.3 \mathrm{c}$ in neutron star candidates in our Galaxy such as Cygnus X-3, SS433, and Crab. Our result and Kudoh \& Shibata (1995) show that the maximum velocity of the jet is order of the Keplerian velocity when the ambient magnetic field is strong enough. Therefore the jet from the black hole may have almost light velocity; $v_{\text {jet }}^{\mathrm{BH}} \sim 2 v_{\mathrm{K}}^{\mathrm{BH}} \sim c$, whereas that of the neutron star may be subrelativistic; $v_{\text {jet }}^{\mathrm{NS}} \sim 2 v_{\mathrm{K}}^{\mathrm{NS}} \sim 0.3 c$. Here, $v_{\mathrm{K}}^{\mathrm{BH}}$ and $v_{\mathrm{K}}^{\mathrm{NS}}$ are Keplerian velocity at the inner disk of the black hole and the neutron star, respectively.

\section{References}

Blandford, R. D. \& Payne, D. G., MNRAS 199, 883 (1983).

Cammenzind, M., in Accretion Disks and Magnetic Fields in Astrophysics, ed. G. Belvedere (Dordrecht, Kluwer 1989), p. 129.

Cao X. \& Spruit H. C., Astronomy and Astrophysics, 287, 80-86 (1994).

Koide, S., Nishikawa, K.-I., \& Mutel, R. L., 1996, ApJ, 463, L71-L74.

Koide, S. 1996, ApJ, submitted.

Kudoh, T. \& Shibata, K., ApJ, 452, L41-L44 (1995).

Li, Z.-Y., Chiueh, T., \& Begelman, M. C., ApJ, 394, 459-471 (1992).

Miley, G. K., 1980, A. Rev. Astr. Astrophys. 18, 165.

Mirabel, I. F. \& Rodriguez, L. F., Nature, 371, 46-48 (1994).

Mirabel, I. F., \& Rodriguez, L. F., Proc. NATO ASI, Solar and Astrophysical MHD Flows, ed. K. C. Tsinganos, (Heraklion, Kluwer 1996), p. 683.

Shibata, K. \& Uchida, Y., Publ. Astron. Soc. Japan, 38, 631-660 (1986).

Tomimatsu, A., Publ. Astron. Soc. Japan, 46, 123-130 (1994).

Acknowledgments. We thank M. Inda-Koide for her discussion and important comments on preliminary version of the manuscript. We also thank K.-I. Nishikawa and R. L. Mutel for their encouragement and also thank the National Institute for Fusion Science and the National Astronomical Observatory for use of super-computers. 


\section{Discussion}

D. Meier: What is the Alfvén velocity (in units of $V_{k o}$ ) initially in the material that is actually ejected? Is only the low density disk atmosphere ejected or is much of the high density ejected?

$S$. Koide: The Alfven velocity at the inner part of the disk is $0.1 V_{k o}$ initially. The low density jet is ejected near the slow point at $3.5 \tau_{k}$. The high density jet is ejected at $4.0 \tau_{k}$ and follows the low density jet. 